\title{
Emergent cosmic space in Rastall theory
}

\author{
Fang-Fang Yuan ${ }^{1}{ }^{*}$, Peng Huang ${ }^{2} \dagger$ \\ ${ }^{1}$ Department of Physics, Hebei Normal University of Science and Technology, \\ Qinhuangdao 066004, China \\ ${ }^{2}$ Department of Information, Zhejiang Chinese Medical University, \\ Hangzhou 310053, China
}

\begin{abstract}
Padmanabhan's emergent cosmic space proposal is exploited to study the Rastall theory which involves modifying the covariant conservation law of energy-momentum tensor. As necessary elements for this approach, we firstly find the Komar energy and the general entropy of apparent horizon in this theory. After that, a modified expansion law is invoked to reobtain the Friedmann equations.
\end{abstract}

\footnotetext{
*kfyuan@yahoo.com

${ }^{\dagger}$ huangp46@mail.sysu.edu.cn
} 


\section{Introduction}

Ever since the groundbreaking research of Jacobson [1, the emergent gravity paradigm has been investigated from various perspectives $[2,3]$. The original idea (from thermodynamics to gravity) is to use the Clausius relation and the properties of local Rindler horizon to derive the Einstein field equation. On the other hand, an inverse approach (from gravity to thermodynamics) has been proposed by Padmanabhan [4 who found that certain component of the gravitational field equations in a static, spherically symmetric spacetime can be rewritten as an ordinary first law of thermodynamics at a black hole horizon. Some aspects of this method are still under investigation [5]

As for the emergent cosmology (from thermodynamics to gravity), Padmanabhan [10] has introduced an intriguing idea that the expansion of the universe can be regarded as a process towards holographic equipartition. This proposal has led to many applications and generalizations, see e.g. [11 16]. In our work, we plan to utilize this approach to study an interesting gravity called Rastall theory [17] which supposes that the energy-momentum is not covariantly conserved in curved spacetimes 1 .

As preparatory steps, we also find the Komar energy in this theory and the general entropy of apparent horizon in the non-flat Friedmann-Robertson-Walker universe. Obviously, these results are important in their own right. As a side note, we would like to mention that this paper is partially inspired by the works in [23,24] which have studied the thermodynamics of apparent horizon in the flat case as well as the relation between Rastall field equation and the first law.

The structure of this work is as follows. In Section 2, we find the Komar energy/mass formula in Rastall theory. In Section 3, we generalize the analysis of [23] to the case of nonflat universe, and find the general entropy of apparent horizon. By starting with a modified expansion law, in Section 4, we apply the emergent cosmic space proposal to Rastall theory and reproduce the Friedmann equations. The conclusion will be given in the last section.

\section{Komar energy in Rastall theory}

Rastall [17] proposed that one fundamental assumption of Einstein's general relativity, i.e. the vanishing of covariant divergence of the energy-momentum, is questionable in curved spacetimes. By supposing a relation as $T_{; \nu}^{\mu \nu}=\lambda R^{, \mu} 2$, a generalization of the gravitational field

\footnotetext{
${ }^{1}$ Over the years, Rastall's idea and formulation have stimulated many investigations [18 22]. Besides the applications in cosmology, Rastall theory can also be related to some modified gravity theories.

${ }^{2}$ We note in passing that Visser's massive graviton model 25] also involves a modification of the energymomentum conservation equation.
} 
equation is found to be

$$
G_{\mu \nu}+k \lambda g_{\mu \nu} R=k T_{\mu \nu} .
$$

Here $G_{\mu \nu}$ is the Einstein tensor and $k$ is the gravitational constant in Rastall theory which is to determined by the consistency with Newtonian limit. The Einstein field equation corresponds to the case where $\lambda=0, k=8 \pi$. Our convention for the Newton's constant is $G=1$.

The Rastall field equation (2.1) can be rewritten as

$$
R_{\mu \nu}=k\left[T_{\mu \nu}-\frac{1-2 k \lambda}{2(1-4 k \lambda)} g_{\mu \nu} T\right] .
$$

According to the standard procedure [26], we see that the Komar energy/mass in Rastall theory has the following form 3 :

$$
E_{K}=\int_{V} d V\left(2 T_{\mu \nu}-\frac{1-2 k \lambda}{1-4 k \lambda} g_{\mu \nu} T\right) u^{\mu} \xi^{\nu} .
$$

Here $u^{\mu}$ is a unit timelike vector and $\xi^{\nu}$ is a normalized Killing vector. Although this is an expected result, we are unaware of any previous detailed study on it in this particular theory.

Considering a perfect fluid again and choosing suitable coordinates, we can define the Komar energy density as (with $E_{K}=\rho_{K} V$ for the usual case)

$$
\begin{aligned}
\rho_{K} & =2 T_{00}-\frac{1-2 k \lambda}{1-4 k \lambda} g_{00} T \\
& =\frac{1}{1-4 k \lambda}[(1-6 k \lambda) \rho+3(1-2 k \lambda) p] .
\end{aligned}
$$

Here $\rho$ is the energy density and $p$ is the pressure. This is the expression we will use in the following discussions. One can also notice that the familiar equation $\rho_{K}=\rho+3 p$ is obviously recovered when $\lambda=0, k=8 \pi$.

\section{Apparent horizon entropy: the non-flat case}

As shown in 23], the method of Cai-Kim [27] can be invoked to obtain the apparent horizon entropy with spatial curvature factor $\kappa=0$. The derivation relies heavily on Hayward's unified first law [28].

We would like to generalize this analysis to the non-flat Friedmann-Robertson-Walker universe. In this case, we can use the Rastall field equation (2.1) to find the Friedmann equations for a perfect fluid as

$$
\begin{aligned}
3(1-4 k \lambda) H^{2}-6 k \lambda \dot{H}+3(1-2 k \lambda) \frac{\kappa}{a^{2}} & =k \rho, \\
3(1-4 k \lambda) H^{2}+2(1-3 k \lambda) \dot{H}+(1-6 k \lambda) \frac{\kappa}{a^{2}} & =-k p .
\end{aligned}
$$

\footnotetext{
${ }^{3}$ One may try to add a prefactor $k / 8 \pi$ in this formula, but it would be inconsistent with our investigation in Section 4
} 
Here $H=\dot{a} / a$ is the Hubble parameter with $a$ the scale factor. The location of the apparent horizon is as usual

$$
r_{A}=\frac{1}{\sqrt{H^{2}+\frac{\kappa}{a^{2}}}} .
$$

The energy flux crossing the apparent horizon assumes a familiar form:

$$
\delta Q=-3 V H(\rho+p) d t .
$$

Using the Clausius relation, the variation of entropy is given by

$$
d S=-\frac{\delta Q}{T}=8 \pi^{2} H r_{A}^{4}(\rho+p) d t .
$$

Recalling the following continuity equation $(\mu=0$ component of the nonconservation relation $\left.T_{; \nu}^{\mu \nu}=\lambda R^{, \mu}\right)$

$$
\frac{1-3 k \lambda}{1-4 k \lambda} \dot{\rho}-\frac{3 k \lambda}{1-4 k \lambda} \dot{p}+3 H(\rho+p)=0
$$

then (3.5) can be rewritten as

$$
\frac{d S}{d t}=-\frac{8 \pi^{2}}{3(1-4 k \lambda)} r_{A}^{4}[(1-3 k \lambda) \dot{\rho}-3 k \lambda \dot{p}] .
$$

Now we insert the Friedmann equations (3.1) and (3.2) into the above expression, and obtain a simple result

$$
\frac{d S}{d t}=-\frac{16 \pi^{2}}{k} H r_{A}^{4}\left(\dot{H}-\frac{\kappa}{a^{2}}\right) .
$$

Noticing that $\dot{r}_{A}=-H r_{A}^{3}\left(\dot{H}-\frac{\kappa}{a^{2}}\right)$, then the apparent horizon entropy is obtained as follows

$$
S=\frac{16 \pi^{2}}{k} \int r_{A} d r_{A}=\frac{8 \pi^{2}}{k} r_{A}^{2} .
$$

This obviously has the familiar form: $S=2 \pi A / k$, where $A=4 \pi r_{A}^{2}$ is the area of apparent horizon 4. Surprisingly, the main result (3.9) of this section is independent of the parameter $\lambda$, and has no entropic correction in Rastall theory.

\section{Friedmann equations from an expansion law}

According to the emergent cosmic space proposal of Padmanabhan [10], the expansion of the universe can be attributed to the difference between the number of degrees of freedom on a

\footnotetext{
${ }^{4}$ Note that the derivation in 23$]$ has an unfortunate mistake (compare (3.9) here with (22) in that reference). This can be most easily seen by inserting the Raychaudhuri equation (9) there into (17) of [23]. In an earlier version of our present work on arXiv, we argued for a modified Komar energy which now turns out to be unnecessary because of this error. Accordingly, a lot of complicated and tedious calculations including an unsuccessful attempt on the non-flat case would have been avoided for an acute reader.
} 
holographic surface $N_{\text {sur }}$ and the one in the bulk $N_{\text {bulk }}$. Thus its expansion is interpreted as a process towards holographic equipartition. In the case of non-flat universe, a modified expansion law has been proposed in [12] as

$$
\frac{d V}{d t}=H r_{A}\left(N_{\text {sur }}-N_{\text {bulk }}\right) .
$$

This is also applicable to the situations with entropic corrections [13].

Using the expression of apparent horizon entropy in (3.9), we can obtain the increase in the effective cosmic volume as

$$
\begin{aligned}
\frac{d V}{d t} & =\frac{r_{A}}{2} \frac{d A}{d t}=2 r_{A} \frac{d S}{d t} \\
& =-\frac{32 \pi^{2}}{k} r_{A}^{5} H\left(\dot{H}-\frac{\kappa}{a^{2}}\right) \\
& =-2 \pi r_{A}^{5} \frac{d}{d t}\left[\frac{8 \pi}{k}\left(\frac{1}{r_{A}^{2}}\right)\right] .
\end{aligned}
$$

This result and the holographic idea invite us to propose the following effective number of degrees of freedom on the apparent horizon

$$
N_{\text {sur }}=4 \pi r_{A}^{2} \cdot \frac{8 \pi}{k}
$$

When $\kappa=0, k=8 \pi$, we have $N_{\text {sur }}=4 \pi / H^{2}$ which corresponds to the standard holographic situation [10] (with the Planck length $L_{P}=1$ ). In this special case, the effective volume reduces to the usual Hubble volume.

Recalling the Komar energy density in (2.4), we find a general formula for the modified number of degrees of freedom in the bulk as

$$
\begin{aligned}
N_{\text {bulk }} & =\frac{2}{T}\left|E_{K}\right|=-2 \rho_{K} \frac{V}{T} \\
& =-\frac{16 \pi^{2}}{3} r_{A}^{4} \cdot \rho_{K} .
\end{aligned}
$$

Here the cosmic volume is $V=4 \pi r_{A}^{3} / 3$, and the Hawking temperature is $T=1 / 2 \pi r_{A}$ [29]. Moreover, the appearance of a minus sign is because we are considering the accelerating phase.

Based on the results in (4.2) and (4.3), we can find that the modified expansion law (4.1) leads to

$$
\rho_{K}=-\frac{3}{16 \pi^{2} r_{A}^{4}}\left[4 \pi r_{A}^{2} \cdot \frac{8 \pi}{k}+\frac{32 \pi^{2}}{k} r_{A}^{4}\left(\dot{H}-\frac{\kappa}{a^{2}}\right)\right] .
$$

Together with the radius of apparent horizon (3.3), we then have an elegant result

$$
\rho_{K}=-\frac{6}{k}\left(H^{2}+\dot{H}\right)
$$


Recalling the detailed formula of the original Komar energy density in (2.4), the above formula enables us to express the pressure as

$$
p=\frac{1}{3(1-2 k \lambda)}\left[-\frac{6}{k}(1-4 k \lambda)\left(H^{2}+\dot{H}\right)-(1-6 k \lambda) \rho\right] .
$$

We then insert this into the continuity equation (3.6). After some manipulations and eliminating a common factor $1 /(1-2 k \lambda)$, we obtain

$$
\dot{\rho}+2 H \rho-\frac{6}{k}(1-4 k \lambda) H\left(H^{2}+\dot{H}\right)+6 \lambda(2 H \dot{H}+\ddot{H})=0 .
$$

Although the appeal to continuity equation may raise some suspicion, it is actually a routine step in this research area. Obviously (3.6) can reduce to a familiar equation as $\dot{\rho}+3 H(\rho+p)=0$.

Multiplying the above equation by $a^{2}$, we obtain

$$
\frac{k}{3(1-4 k \lambda)}\left(a^{2}+2 a \dot{a} \rho\right)=2 a^{2} H\left(H^{2}-\frac{2 k \lambda}{1-4 k \lambda} \dot{H}\right)+a^{2}\left(2 H \dot{H}-\frac{2 k \lambda}{1-4 k \lambda} \ddot{H}\right) .
$$

This can be rewritten as

$$
\frac{k}{3(1-4 k \lambda)} \frac{d}{d t}\left(\rho a^{2}\right)=\frac{d}{d t}\left[a^{2}\left(H^{2}-\frac{2 k \lambda}{1-4 k \lambda} \dot{H}\right)+C\right] .
$$

Here we have an integration constant $C$ which is not necessarily zero. Through the comparison with the Friedmann equation (3.1), one can inspect that by adjusting the arbitrary constant $C$, these two equations agree even when the curvature factor $\kappa \neq 0$. We note in passing that to derive this result, one may also use the invariant volume instead of the above aerial volume as in [14].

To sum up, in the context of an unordinary gravity like Rastall theory, the basic expansion law can still lead to the fundamental equations for the expansion of universe.

\section{Conclusion}

In this work, we firstly propose a Komar energy density (2.4) for a perfect fluid in Rastall theory. After that, we generalize the study in [23] to the non-flat universe, and find the entropy of apparent horizon in (3.9). Based on these analyses, we then apply a modified expansion law (4.1) in the emergent cosmic space proposal to Rastall theory, and recover the basic Friedmann equation in (3.1). Because of an arbitrary integration constant, the derivation here is independent of the value of curvature factor. Notice also that the consistency for the case with $r_{A} \neq 1 / H$ requires us to use a modified expansion law like (4.1).

As for further investigations, it would be valuable to extend this work to the Brans-DickeRastall theory [21]. Secondly, one may consider the case of event horizon in Rastall theory along the line of [30,31]. Thirdly, [24] has recently shown the relation between Rastall field 
equation and the ordinary first law of thermodynamics. By using the method of [5, 6], maybe the exact first law of black hole mechanics (with black hole charges) could also be obtained in this theory. Finally, the idea that the energy-momentum is not covariantly conserved in cosmology perhaps could be combined with the recent proposal of uniformly expanding vacuum [32].

\section{Acknowledgments}

We are indebted to an anonymous referee for valuable and meticulous suggestions. FFY is partially supported by the Doctoral Foundation of HNUST; PH is supported by the Doctoral Foundation of ZCMU.

\section{References}

[1] T. Jacobson, Phys. Rev. Lett. 75 (1995) 1260

[2] E. P. Verlinde, JHEP 1104 (2011) 029

[3] T. Faulkner, M. Guica, T. Hartman, R. C. Myers and M. Van Raamsdonk, JHEP 1403 (2014) 051

[4] T. Padmanabhan, Class. Quant. Grav. 19 (2002) 5387

[5] Y. Kwon and S. Nam, arXiv:1310.4933 [gr-qc]

[6] Y. G. Miao, F. F. Yuan and Z. Z. Zhang, Int. J. Mod. Phys. D 23 (2014) no.11, 1450093

[7] S. Chakraborty and T. Padmanabhan, Phys. Rev. D 90 (2014) no.8, 084021

[8] S. Chakraborty, K. Parattu and T. Padmanabhan, JHEP 1510 (2015) 097

[9] S. Chakraborty and T. Padmanabhan, Phys. Rev. D 92 (2015) no.10, 104011

[10] T. Padmanabhan, arXiv:1206.4916 [hep-th]

[11] R. -G. Cai, JHEP 1211 (2012) 016

[12] A. Sheykhi, Phys. Rev. D 87 (2013) no.6, 061501

[13] F. -F. Yuan and Y. -C. Huang, arXiv:1304.7949 [gr-qc]

[14] E. Chang-Young and D. Lee, JHEP 1404 (2014) 125

[15] A. Sepehri, F. Rahaman, A. Pradhan and I. H. Sardar, Phys. Lett. B 741 (2015) 92 
[16] A. Sepehri, F. Rahaman, S. Capozziello, A. F. Ali and A. Pradhan, Eur. Phys. J. C 76 (2016) no.5, 231

[17] P. Rastall, Phys. Rev. D 6 (1972) 3357

[18] L. L. Smalley, Phys. Rev. D 9 (1974) 1635

[19] J. C. Fabris, M. H. Daouda and O. F. Piattella, Phys. Lett. B 711 (2012) 232

[20] E. R. Bezerra de Mello, J. C. Fabris and B. Hartmann, Class. Quant. Grav. 32 (2015) no.8, 085009

[21] T. R. P. Carams, M. H. Daouda, J. C. Fabris, A. M. Oliveira, O. F. Piattella and V. Strokov, Eur. Phys. J. C 74 (2014) no.11, 3145

[22] I. G. Salako, M. J. S. Houndjo and A. Jawad, Int. J. Mod. Phys. D 25 (2016) no.07, 1650076

[23] H. Moradpour, Phys. Lett. B 757 (2016) 187

[24] H. Moradpour and I. G. Salako, arXiv:1606.06589 [gr-qc]

[25] M. Visser, Gen. Rel. Grav. 30 (1998) 1717

[26] A. Komar, Phys. Rev. 113 (1959) 934

[27] R. -G. Cai and S. P. Kim, JHEP 0502 (2005) 050

[28] S. A. Hayward, Class. Quant. Grav. 15 (1998) 3147

[29] R. -G. Cai, L. -M. Cao and Y. -P. Hu, Class. Quant. Grav. 26 (2009) 155018

[30] S. Saha and S. Chakraborty, Phys. Lett. B 717 (2012) 319

[31] S. Mitra, S. Saha and S. Chakraborty, Phys. Lett. B 734 (2014) 173

[32] P. Huang and F. -F. Yuan, Phys. Dark Univ. 12 (2016) 45 